Supporting Information

\title{
Porous Composite Gel Polymer Electrolyte with Interfacial Transport Pathways for Flexible Quasi Solid Lithium-Ion Batteries
}

Yanjun $\mathrm{Xu}^{\dagger \mathrm{a}}$, Lina $\mathrm{Gao}^{\dagger \mathrm{b}}$, Xianzhang $\mathrm{Wu}^{\mathrm{c}}$, Shengzhao Zhang ${ }^{\mathrm{a}}$, Xiuli Wang*a, Changdong $\mathrm{Gu}^{\mathrm{a}}$, Xinhui Xia ${ }^{\mathrm{a}}$, Xueqian Kong ${ }^{* \mathrm{~b}}$ and Jiangping $\mathrm{Tu}^{* \mathrm{a}}$

a. State Key Laboratory of Silicon Materials, Key Laboratory of Advanced Materials and Applications for Batteries of Zhejiang Province, and School of Materials Science and Engineering, Zhejiang University, Hangzhou, 310027, China.

b. Center for Chemistry of High-Performance \& Novel Materials, Department of Chemistry, Zhejiang University, Hangzhou, 310027, China.

c. Research Institute of Narada Power Source Co. Ltd, Hangzhou 310012, China.

$\dagger$ These authors contributed equally to this work.

* Corresponding author. Tel.: +86 571 87952573; Fax: +86 57187952856.

E-mail: wangxl@zju.edu.cn (X.L. Wang); kxq@zju.edu.cn (X.Q. Kong); tujp@zju.edu.cn (J.P. Tu). 

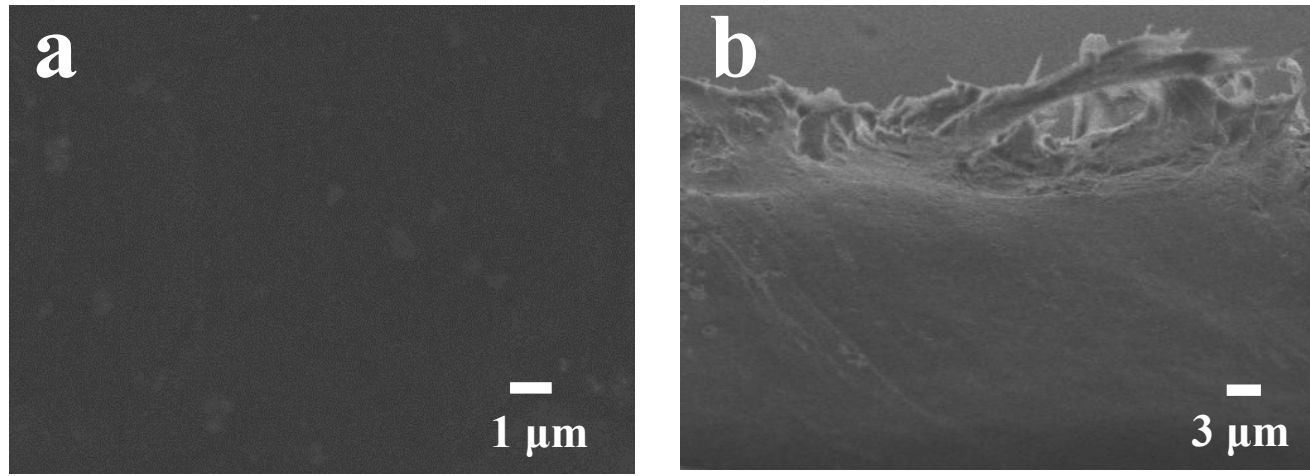

Figure S1. SEM images of DCGPE membrane: (a) surface and (b) cross-section.

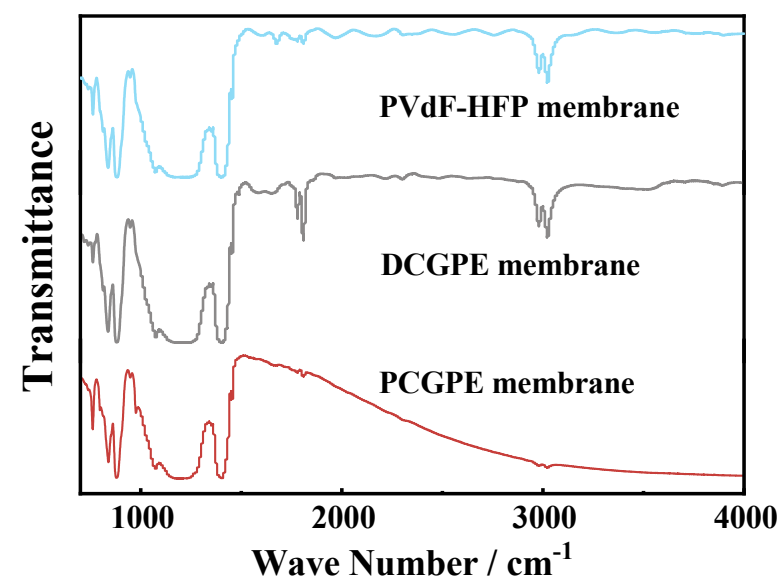

Figure S2. FTIR spectra of PCGPE, DCGPE and PVdF-HFP membranes.
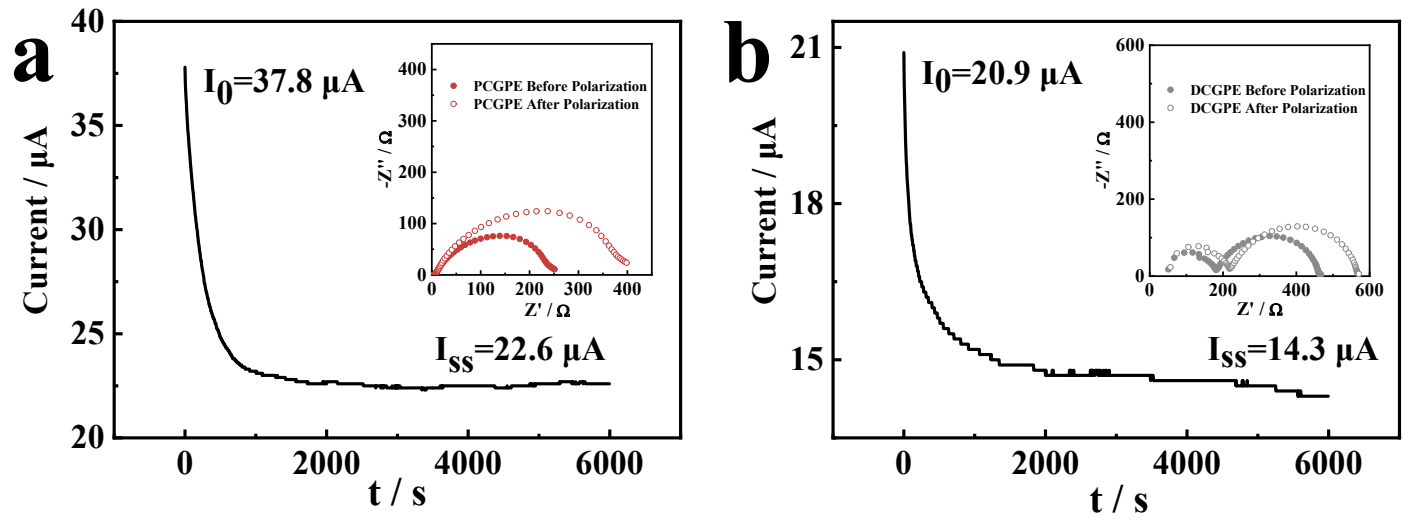

Figure S3. Current-time plots tested by chronoamperometry at a DC polarization of $0.01 \mathrm{~V}$, inset: EIS response before and after the polarization: (a) PCGPE, (b) DCGPE, $I_{0}$ : the initial current, $I_{s s}$ : the steady current. 


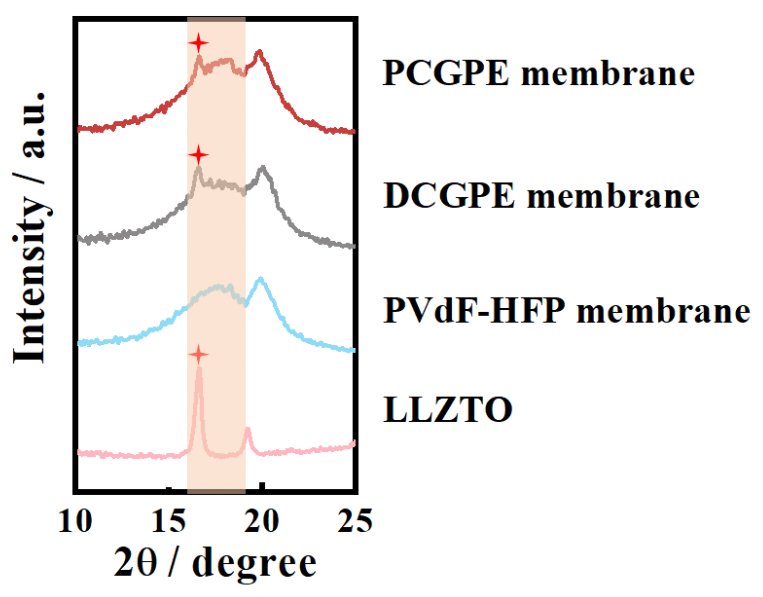

Figure S4. Enlarged XRD patterns of PCGPE, DCGPE, PVdF-HFP membranes and LLZTO. $2 \theta$ degree of the star marked position is $16.6^{\circ}$.

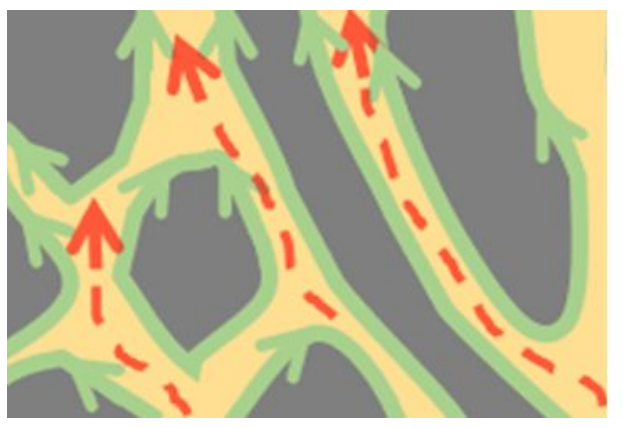

\section{PVdF-HFP/LLZTO matrix}

\section{Pores in the matrix}

Fast $\mathrm{Li}^{+}$pathways on porous interfaces

Slow $\mathrm{Li}^{+}$pathways in the matrix

Figure S5. Schematic diagram of $\mathrm{Li}^{+}$pathways in PCGPE. 